\title{
ON THE DESIGN OF BROADBAND QUASIOPTICAL SYSTEMS FOR SUBMILLIMETER-WAVE RADIO-ASTRONOMY RECEIVERS
}

\author{
S. Withington, ${ }^{1}$ J. A. Murphy, ${ }^{2}$ A. Egan, ${ }^{2}$ and R. E. Hills ${ }^{1}$ \\ ${ }^{1}$ Cavendish Laboratory, Madingley Road \\ Cambridge, England \\ ${ }^{2}$ St. Patrick's College, Co. Kildare \\ Maynooth, Ireland
}

Received June 3, 1992

Abstract: In this paper, we summarize the issues that should be considered. when designing broadband quasioptical systems for submillimetre-wave radioastronomy recєivers. We cover topics such as bandwidth, cross-talk, truncation, and aberrations, and we argue that it should be possible to manufacture high-efficiency systems that have several octaves of bandwidth. A key feature of the paper is that we use the language of multimode Gaussian optics throughout, and in this way, we emphasize that a receiver is a diffractionlimited imaging system rather than just a collection of components for guiding Gaussian beams. The whole discussion is conducted in terms of a particular system we are constructing for the James Clerk Maxwell. Telescope in Hawaii.

\section{Introduction}

In this paper, we discuss the design of broadband quasioptical systems for submillimetre-wave radio-astronomy receivers. We cover topics such as bandwidth, cross-talk, truncation, and aberrations, and we argue that it should be possible to manufacture high efficiency systems that have several octaves of bandwidth. A key feature of the paper is that we use the language of multimode Gaussian optics throughout, and in this way we emphasize that: a receiver is a diffraction-limited imaging system, rather than just a collection of components for guiding Gaussian beams. The whole discussion is conducted in terms of a particular system we are constructing for the James 
Clerk Maxwell Telescope (JCMT) in Hawaii. The system combines the functions of imaging, local oscillator injection, sideband filtering, and calibration [1], and in principle, it can be used anywhere in the frequency range $200 \mathrm{GHz}$ to $900 \mathrm{GHz}$ without changing horns or mirrors. The system can be configured to operate in a dual-frequency mode, where one observes two spectral lines at different frequencies simultaneously, or in a dual-polarization mode, where one observes a single spectral line in two orthogonal linear polarizations simultaneously. It is straightforward to switch between the two configurations, and either local oscillator source can be used to drive the mixers when the receiver is operating in the dual-polarization mode.

\section{Basic Design}

The optics of a submillimetre-wave heterodyne receiver must image the fields at the aperture of the telescope onto the mouth of the horn. Ideally, this coupling should be independent of frequency, not only over the tuning range of a particular mixer, but over the full range of frequencies likely to be observed by the telescope. We are attempting to build an achromatic optical system, for the JCMT, that can operate anywhere in the frequency range $200 \mathrm{GHz}$ to $900 \mathrm{GHz}$. In this way, we hope to use the same basic receiver for all of the submillimetre-wave atmospheric windows. For a correctly designed system, the low-frequency limit is set by truncation in the optics, and the high-frequency limit is set by surface roughness and mechanical stability. The aberrations caused by using off-axis mirrors at frequencies away from the nominal design frequency are a potential problem also.

It is desirable, although by no means necessary, for a radio-astronomy receiver to be able to observe in two orthogonal polarizations simultaneously. In this way, the observing time required to achieve a given sensitivity can be reduced by a factor of two. Given that a dual-polarization receiver has two independent channels, it is also desirable to keep open the possibility of running the receiver in a dual-frequency mode. In the dual-frequency mode one observes two spectral lines in different atmospheric windows simultaneously, thereby increasing the spectral throughput of the telescope during periods of high atmospheric transmission. Dual-frequency observing should make pointing at the shortest wavelengths more straightforward, and it also should enable the relative positions and intensities of lines to be determined more accurately than would be the case for separate observations.

We have devised a simple scheme that enables a receiver to be used for either dual-frequency or dual-polarization work, and it is straightforward to switch between the two configurations. The basic idea is to create a dualchannel instrument by splitting the incoming radiation into two orthogonal 
linear polarizations, and thereafter having two nearly independent receivers for down converting the two beams. It is necessary, however, to configure the optics in such a way that a single local oscillator source can inject power, in the appropriate polarizations, into both channel simultaneously, thereby producing a dual polarization instrument. Initially, we considered a whole range of schemes for sharing optical components between channels, however because it is necessary to keep cross-talk to a minimum, we abandoned these elegant schemes in favour of a more conservative design.

After considering many different designs, we chose the arrangement shown in Fig. 1, where Martin-Puplett interferometers are used for both local oscillator injection and for single-sideband filtering. If we were only interested in building a dual-polarization receiver we would only need to pass the signal beam through one interferometer, however, because we wish to preserve the dual-frequency capability, we need to pass the signal beam through two interferometers, and the insertion loss of the optics is increased by a small amount.

The first part of the optical system comprises two chopper wheels, CH1(B) and $\mathrm{CH} 2(\mathrm{~B})$, for switching the input beam between hot and cold loads. The image band appears at a separate port, and this beam can also be switched, CH1(A) and CH2(A), between hot and cold loads for performing sideband measurements. During normal operation the image will, of course, be terminated in a cryogenic load. In order to reduce the heat load on the Dewar, the design shown in Fig. 1 uses only one image load.

The input grid, G1, separates the incoming radiation into two orthogonal polarizations, and thereafter, the two polarizations are kept completely separate and down converted by essentially two different receivers. The first interferometer after the input grid acts as a single-sideband filter. Each channel then has its own grid, G3(A) and G3(B), for injecting local oscillator power. Fig. 1 shows the receiver operating in the dual-frequency mode, in that each channel has its own local oscillator source. It is possible, however, by rotating one of the grids, to inject power from either source into both channels simultaneously, thereby creating a dual-polarization receiver. It is necessary, to rotate the polarization of the appropriate source by $45^{\circ}$, and a half-wave plate [3] is provided for this purpose. An important feature of the above design is that polarization splitting, rather than amplitude splitting, is used throughout. Wire grids can be made to behave as almost ideal polarizers over several octaves [4], whereas partially reflecting surfaces tend to be frequency dependent. Furthermore, it is very difficult to make wellmatched pairs of amplitude-splitting meshes, and asymmetries in dual-beam interferometers cause increased insertion loss and reduced visibility.

A further issue worth mentioning at this stage is the single-sideband filter. 


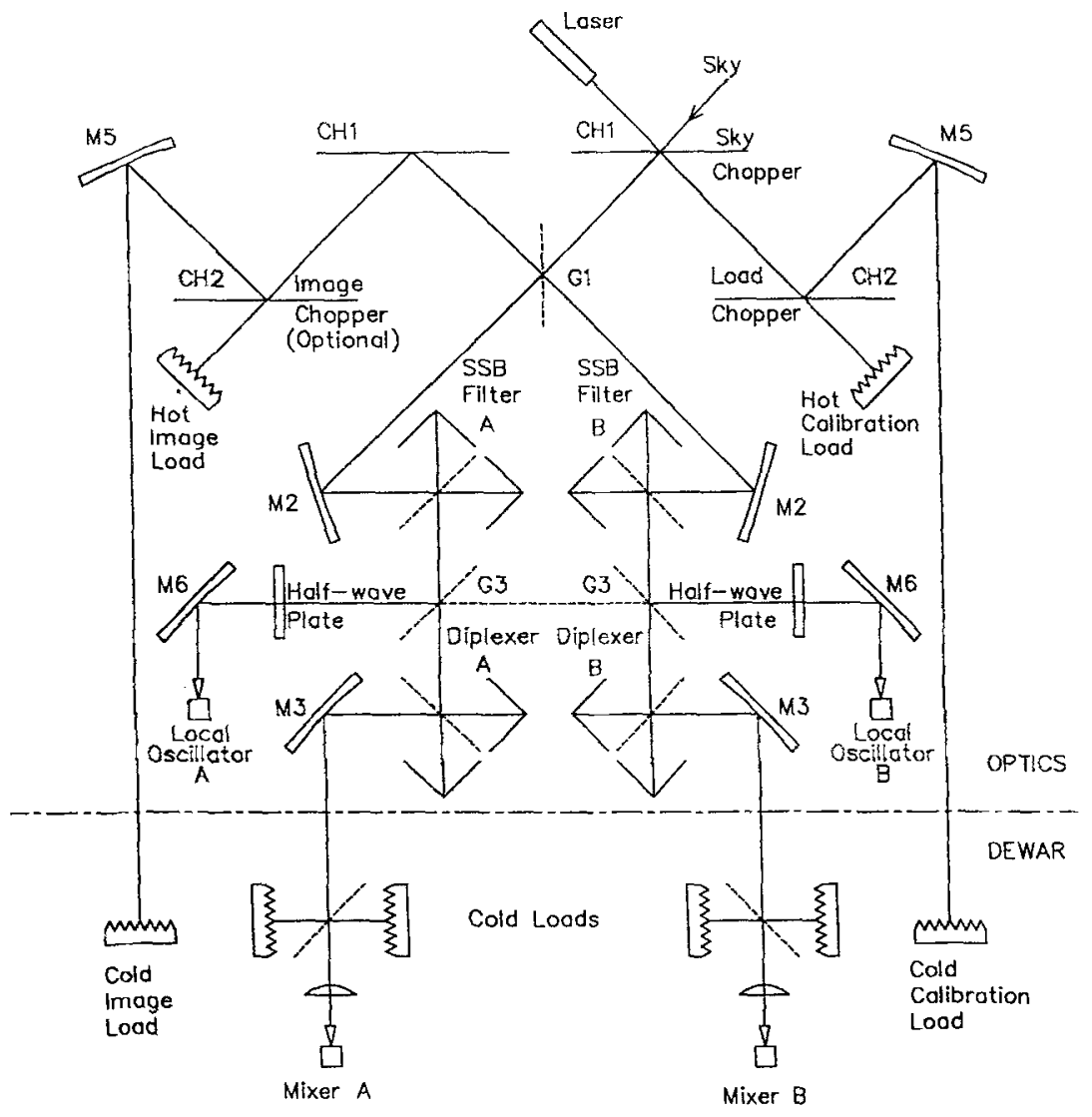

Figure 1: A block diagram of the broadband receiver. 
At low frequencies, the principal function of the filter is to prevent confusing spectral lines being down converted from the image band, however, at short submillimetre wavelengths, the single-sideband filter has two other important roles. First, the atmospheric transmission in the short-wavelength windows has a great deal of steep-sided structure on a frequenry scale comparable with the sideband spacing, and it will not be possible to make well-calibrated measurements of spectral-line sources unless the image response is suppressed. Second, if the image is terminated through atmospheric absorption, rather than on the cold sky, the noise entering the image will increase the overall system noise temperature. Although the mixer may offer some degree of image rejection, complete isolation cannot be relied upon, and it is almost certain that the sideband ratio will vary across the RF band. Moreover, the present trend in SIS mixer design is to tune out the capacitance of the tunnel junction with integrated transmission lines [2], and future generations of mixers will probably not have any mechanical tuning at all. Given that SIS mixers work well when their sidebands are terminated with equal impedances, we take the view that it is best to design the mixers for double-sideband operation, and to use quasioptical techniques for resistively terminating the image in a cold load.

Because local osillator power is difficult to generate at high frequencies, we prefer not to rely on Mylar beam splitters for dual-polarization work at short submillimetre wavelengths. The above arrangement places the singlesideband filter ahead of the diplexer so that $50 \%$ of the local oscillator power is not lost in the image load. In passing, it is probably worth mentioning that we also considered using Fabry-Perot interferometers to inject the local oscillator power. Unfortunately, Fabry-Perot interferometers have a number of disadvantages. First, in simple parallel-mesh designs, the partial reflectors must remain flat and parallel to within a fraction of a wavelength as the interferometer is tuned. Second, the separation of the reflectors determines the intermediate frequency, and because the beam enters at $45^{\circ}$, it is not possible to use a reasonable frequency without incurring problems with either beam clearance or walk-off. Third, it is necessary to select the reflectivity of the meshes, and this will vary widely with frequency. Finally, the signal comes off the interferometer in reflection, and in order to keep system losses to a minimum, it is necessary to use an interferometer with a sharply-peaked transmission characteristic. Because the local oscillator signal is coupled in through this high-Q filter, there is a danger, if the interferometer is not tuned correctly, that the local oscillator power level will vary as the receiver is tippei. A secondary, but nevertheless important, consideration is that the optics should not lead to frequency-dependent pointing; correctlyaligned Martin-Puplett interferometers have the advantage that the paths 
of the input and output beams do not vary as the interferometer is tuned. Martin-Puplett interferometers do, however, have a sinusoidal pass band, and therefore, the bandwidth is limited to about $1 \mathrm{GHz}$ for an intermediate frequency of $4 \mathrm{GHz}$.

Later, we will show that if the sideband filter and the diplexer are placed the other way round, there is a potential problem with cross talk. Clearly, if we hope to use the receiver for dual-frequency work, we must be careful to maintain a high level of isolation between the two channels. With the sideband filter and diplexer the "correct" way round, cross-talk can only occur through cross-polar scattering, and it should be possible to build a receiver with a high level of isolation. Having only one image load operating in two different polarizations does mean that we cannot use a Brewsterangle window to reduce reflections. Furthermore, because we wish to support dual-frequency observations, we cannot use a resonant window. Co-polar reflections at the image load will generate standing waves which will modulate the effective sideband temperature, and co-polar reflections at the calibration load will modulate the effective calibration temperature. In both cases, we will reduce the effects of standing waves on the long back-coupled paths by tilting the windows, and we will calibrate the signal load by using a laboratory standard.

To minimize losses and to avoid problems with complex standing waves, we have used $90^{\circ}$ off-axis reflective optics throughout. The only exception is the first lens after the mixer. This lens not only corrects for the phase error across the mouth of the horn, but also reduces the radiative heat load on the detector. An important point to note is that although the optics look complicated, there are only two curved mirrors in the signal path of each channel. Several reflections occur off flat surfaces, however, the flat surfaces can be lapped, and the scattering losses made very small. Most of the loss will occur at the curved surfaces. To keep the total loss in each channel below $2 \%$, at $500 \mathrm{GHz}$, requires the surface roughness of each curved mirror to be better than $5 \mu \mathrm{m} \mathrm{rms}$; it should be possible to manufacture such mirrors on a numerically-controlled machine.

\section{System layout}

The layout shown in Fig. 2 and Fig. 3 has a number of important features.

Apart from any transmission asymmetries in the input polarizing grid, the two channels are identical, and therefore it should be straightforward to align the channels and compare their performances. During the construction of previous instruments, we found it very useful to compare performances in this way, and we discovered a number of subtle problems that would have gone 


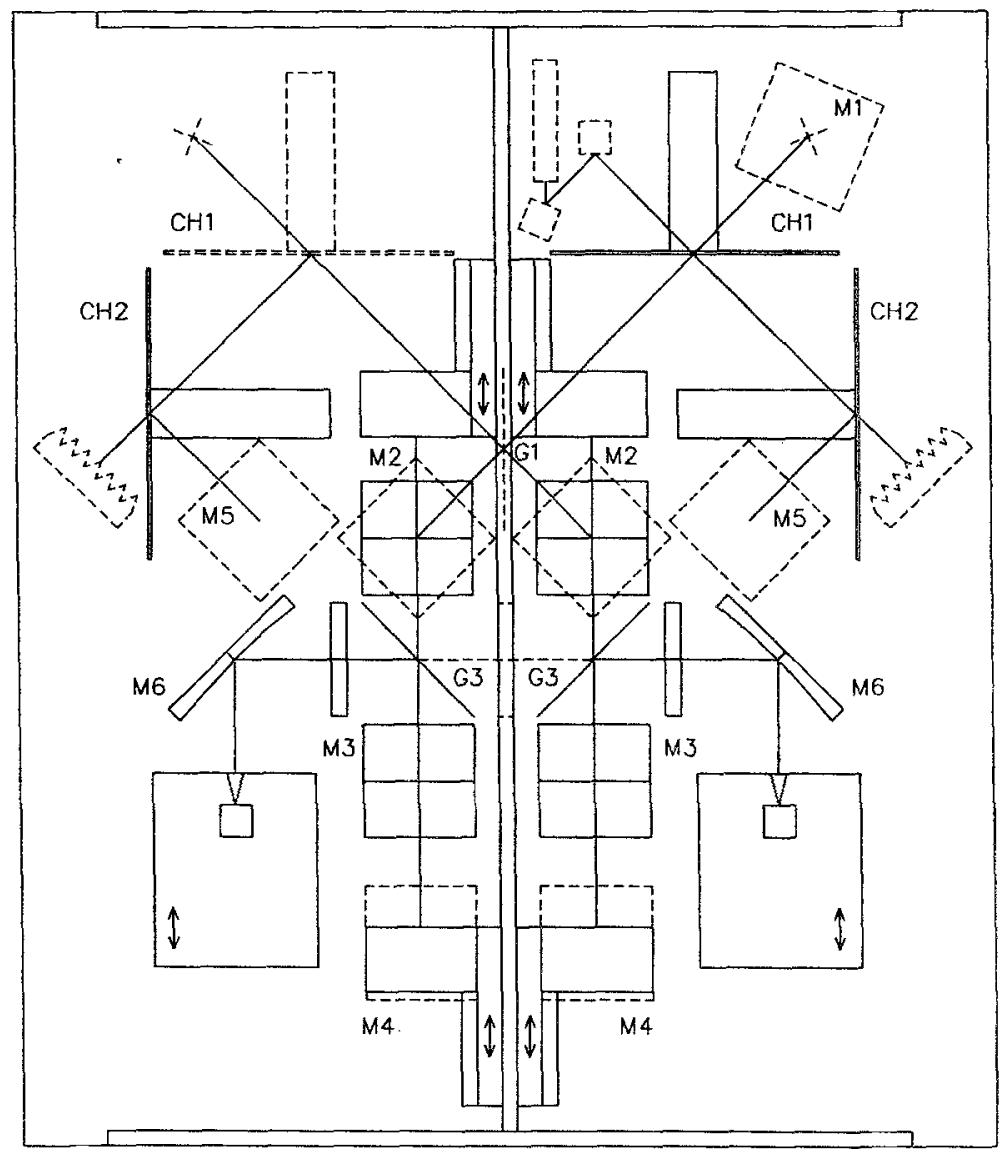

Channel A

Channel B

Figure 2: Front view of the receiver. The input beam enters perpendicularly to the plane of the paper, and is incident on the flat alignment mirror M1. 


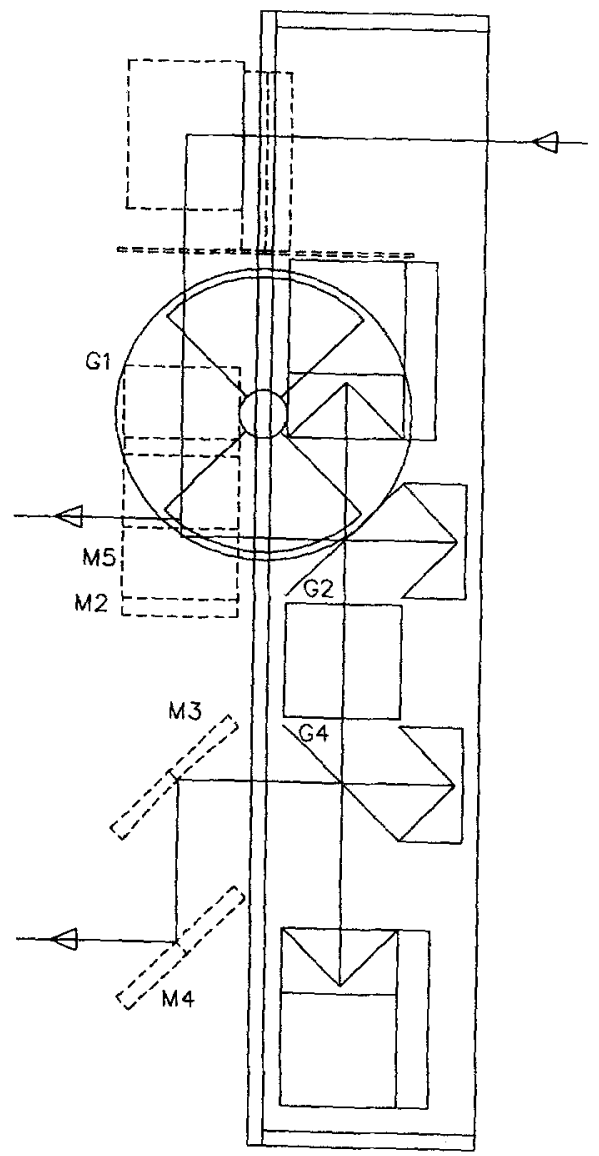

Figure 3: Side view of the receiver. 
unnoticed in a single-channel receiver. Most of the optics associated with the two channels are separated by a single, large aluminium plate which protrudes from the main optical table. This plate leads to a rigid construction, which is of course necessary at very short wavelengths. The narrowest beam in the receiver, ignoring the virtual waist in the horn, is a few millimeters in diameter, and therefore it should be relatively straightforward to keep angular and lateral movements, as the telescope is moved in elevation, well within the implied limits. In practice, the main problem is likely to come from longitudinal movement because, although the optics have a large depth of focus, longitudinal movements will modulate the standing waves on the local oscillator path. To keep the design as compact as possible we have chosen to move only one mirror on each of the interferometers. This constraint reduces the possibility of mechanical instability, but it will now not be possible to compensate for the effects of standing waves by varying the absolute path lengths through the interferometers.

During the development of previous receivers, we found that a significant amount of diffraction can occur around the edges of optical components, which have, in principle, been designed to be very much larger than the size of the beam. In a single-frequency receiver, these losses are small enough to be neglected, but in a dual-frequency receiver these losses may be sufficient to allow local oscillator power from one channel to leak into the other. Crosstalk will be discussed later, however, for the moment it is sufficient to say that the present design minimizes the effects of beam leakage by having a large metal plate between the two channels.

One of the aims of our work has been to make the optics frequency independent. That is to say it should be possible to operate the receiver in any of the submillimetre-wave atmospheric windows without changing the optical system at all. In principle, this flexibility can be achieved by producing a frequency-independent image of the aperture of telescope at the mouth of the horn [5]. In practice, the degree to which an optical system can be made frequency independent has yet to be established. The design shown in Fig 2 is frequency independent in that there is a curved image of the telescope at the mouth of the horn; this curvature is necessary to compensate for the finite length of the horn and is achieved by adjusting the parameters of the last lens. An important point is that the phase-error compensation is the same for all types of horn, diagonal, corrugated, Potter, etc., and the type of horn simply determines the maximum efficiency achieved.

A difficulty with the present design is that one ends up with a diffractionlimited image of the sky at the Dewar window, and yet one wants to minimize the size of the window without truncating the beam. To get an image of the horn at the window it would be necessary to put an extra mirror in the Dewar. 
Not only would it be difficult to align the mirror, but one would not be left with sufficient degrees of freedom external to the Dewar to allow pointing and collimation adjustments with the receiver cold. We have, therefore, been careful to keep the characteristic beam size at the first focus as small as possible. The cold load windows are more straightforward to design as there is an image of the aperture at the Dewar wall.

A further problem, and one which is particularly difficult to circumvent, is that, although the coupling to the telescope is optimum, the coupling to the local oscillator horn is not. The problem occurs because the local oscillator horn is not imaged onto the mixer. The loss of power, which is of the order of $10 \%$, is not particularly important in a single-frequency receiver, but in a dual-frequency system, one has to worry about where the uncoupled power ends up: one does not want a large amount of stray power bouncing around inside the Dewar. In our design, we have chosen to use a Potter horn for the $500 \mathrm{GHz}$ local oscillator source. A Potter horn produces an almost perfect Gaussian beam, and one only has to worry about the lowest order Gaussian mode when designing the optics. This approach leads to a compact design requiring only one off-axis mirror; unfortunately, it does not solve the coupling problem.

Consider for a moment how the optics can be converted from a dualfrequency to dual-polarization system. Fig. 2 shows that local oscillator power can be introduced, from one source, into both channels by opening up a port in the wall dividing the two channels, and rearranging one of the local oscillator injection grids. The large depth of focus means that power balance is not a problem even though one of the beams has to travel farther. The need to keep the coupling equal is also helped by the local oscillator beam being Gaussian. An additional constraint on the layout is that we wish to keep the local oscillator polarization orthogonal to the bottom optics plate so that the local oscillator sources are easy to mount. Also, we want to avoid having to rotate the grids in the interferometers, or any other components, as then the assembly would be much larger. The solution is to have one of the injection grids with its wires perpendicular to the main optics plate, and the other with its wires parallel to the main optics plate. For dual-polarization work we can then use a half-wave plate to rotate the local oscillator signal by $45^{\circ}$ so that half of the power goes into each channel. The half-wave plate can also be used for balancing local oscillator power between the channels, and in the dual-frequency mode, the half-wave plate can be used as a quasioptical attenuator.

It is of course important to ensure that the design has sufficient degrees of freedom to allow collimation and pointing adjustments whilst the receiver is cold. Basically, one needs to produce a variable phase slope across an image 
of the aperture in order to align pointing, and a variable phase slope across an image of the sky in order to align collimation. It must be possible to adjust these for each Cartesian coordinate and for each channel separately. Moreover, one does not want to upset the alignment when installing the receiver on the telescope, and so the first element is a single flat collimating mirror, M1, which is common to both channels. To help installation, we incorporate a laser whose beam comes off the back of the sky chopper, and this beam is aligned with the radio beams before the receiver leaves the laboratory.

\section{Cross-talk}

One of the recurrent themes of this paper has been the desire to keep the cross-channel coupling to a minimum, and it is worth considering this point further. In general, the optics should be large enough to prevent power being scattered off the edges of optical components. Unfortunately, when horns with highly-truncated aperture fields are used, such as diagonal horns, it is very difficult to reduce the truncation, at certain planes, below $1 \%$. To avoid cross-talk through edge effects, we have, therefore, inserted a large metal plate between the two channels. Even with perfect screening, however, there is still the possibility of power being coupled through the well-focused, collimated beams.

In general, one cannot assume that there is any particular relationship between the observing frequency of one channel and the local oscillator frequency of the other: one cannot assume that the interferometers are in any particular position. The LO power incident on the diplexer is coupled into the two output polarizations in different amounts depending on the positions of the mirrors. These waves can then be scattered off the mixer, in either a co-polar or cross-polar sense, and transmitted back through the interferometer. The power that receives a net co-polar scattering will travel back towards the local oscillator source and will result in a standing wave which modulates the power from the source, whereas the power that receives a net cross-polar scattering will travel out along the signal path towards the single-sideband filter, and a proportion of it may be coupled into the other channel. It is straightforward to show that the maximum LO-to-signal reflection coefficient allowed by the interferometer from cross-polar scattering at the mixer is $0 \mathrm{~dB}$, whereas that allowed for co-polar scattering is $-3 \mathrm{~dB}$. Hence, for co-polar scattering at the mixer there is always a $3 \mathrm{~dB}$ reflection loss in the interferometer. If one is only interested in the scattering of power at the fundamental frequency, then power scattered off the mixer in a co-polar sense will always end up back in the LO, and none will be cross 
coupled, whereas power scattered off the mixer in a cross-polar sense will always end up back in the signal beam. Hence, although co-polar reflections off the mixer and Dewar window are difficult to avoid, it should be possible to minimize cross-polar scattering and almost eliminate cross-talk. For example, by placing a cooled polarizing grid close to the Dewar window, any cross-polar scattering off the mixer, lens, or Dewar can be absorbed in a cold load. Now, any power incident on the single-sideband filter gets split between the image load and the sky and calibration loads. The net effect, however, is the same regardless of where the power is reflected; at the fundamental frequency, half of the power goes each way. The important point is that, once again, only cross-polar scattering from the loads will contribute to cross-talk, and therefore two cross-polar scattering events are needed before power can get from one channel into the other. Consequently, with well-designed loads it should be possible to keep cross-talk to a low level even if significant copolar reflection occurs. Cross-polar scattering at the mirrors is ayoided by using long-focal-length optics.

\section{Detailed design}

We have chosen to design the system for corrugated horns in the frequency range $200 \mathrm{GHz}$ to $400 \mathrm{GHz}$ and diagonal horns [6] in the frequency range $400 \mathrm{GHz}$ to $900 \mathrm{GHz}$; of course, corrugated horns can be used at the higher frequencies if they can be manufactured. Diagonal horns are easy to manufacture, and in principle, they can achieve aperture efficiencies of around $82 \%$ if positioned correctly. Although the aperture efficiencies of corrugated and diagonal horns are similar, their loss mechanisms are very different. Corrugated horns loose efficiency because their aperture fields are tapered, whereas diagonal horns loose efficiency because power is coupled into the cross-polar field. As long as one can make a sufficiently large optical system, the power lost when using a diagonal horn can be terminated in a well-controlled way by using a polarizing grid and a cold load.

When designing receivers, we take the view that it is best to optimise the aperture efficiency of the telescope rather than the beam efficiency. Clearly, when observing point sources the coupling to the source is greatest, and the sensitivity is best, when the aperture efficiency is maximized. When mapping extended sources the resolution is greatest when the aperture efficiency is maximized, but sidelobe structure confuses the map. This structure can, however, be removed by smoothing or deconvolution techniques, such as maximum entropy, and in both cases, the final sensitivity is better than if the beam illuminating the telescope had been smoothed. A further advantage of maximizing aperture efficiency is that one can make the illumination 
frequency independent.

The details of the optical design are summarized in Table 1. To achieve frequency independence, the focusing mirrors are separated by the sum of their focal lengths, and to achieve maximum aperture efficiency, the image is formed on a curved surface. We have chosen to curve the image by using the lens in the Dewar. This approach keeps the point at which the fields are most diffuse coupled to the smallest waists, and the points at which the fields have finite spatial extent coupled to the largest waists. In this way, we minimize the size of the optics. The problem with this approach is that the distance between the lens and M3 becomes rather large, and the mechanical design must be able to accomodate this path. The effect of not correcting for the phase error across the mouth of the horn can be seen in the plots of Padman [5] and Withington [6]. The aperture efficiency is maximized when the horn is coupled to an image of the telescope. If the horn is coupled to an appropriately scaled Fourier transform of the image, the maximum aperture efficiency is reduced by about $8 \%$ in the case of a diagonal horn and by about $4 \%$ in the case of a corrugated horn. Usually, however, one finds oneself in an intermediate situation where the aperture efficiency is reduced by as much as $21 \%$ in the case of a diagonal horn and by $13 \%$ in the case of a corrugated horn. Clearly, in this respect, and in a number of others, diagonal horns are less tolerant of bad optical design than are corrugated horns.

\section{Truncation}

Corrugated horns are also easier to use than diagonal horns from a truncation point of view. Corrugated horns have a tapered aperture distribution, and therefore, their far-field beam patterns are also tapered. In fact, full multimode, as opposed to single-mode, calculations show that, even in the far field, almost all of the power is contained within three beam waists. This observation confirms the rule of thumb that optics should be large enough to accommodate three beam radii. In the case of a diagonal horn, however, the aperture field is highly truncated, and the far-field beam has a great deal of side-lobe structure. Clearly, one needs a rigorous way of determining at what radius such a beam can be truncated.

We have developed a series of contour plots that enable truncation calculations to be done for a number of different types of horns. These plots are easy to use but difficult to generate, and so here we simply use the appropriate plot for the co-polar fields of a diagonal horn, and we leave a detailed description of the technique to another paper [7]. The scheme is based on the notion that the power distribution across any plane within a beam is completely determined by the local beam-width parameter, $\omega(z)$, and the 
phase slippage between Gaussian modes. That is to say, the form of the field distribution at a plane is determined by the phase slippage, and the size of the distribution is determined by the width parameter. The variation of the beam width with distance characterizes the way in which the scale size of the beam propagates, whereas the variation of the phase slippage characterizes the way in which the image propagates. Hence, we can calculate the fraction of the power transmitted by a stop, by integrating the power density out to some normalized radius, and we can plot the loss as a function of phase slippage. In Fig. 4, we show contours of constant loss, as a function phase slippage, for the co-polar fields of a diagonal horn. A phase slippage of zero corresponds to an image of the aperture of the horn, and the power at that plane is well confined. A phase slippage of $90^{\circ}$, on the other hand, corresponds to an image of the far field, and the power is very diffuse. It is important to realize that the central portion of this plot does not necessarily correspond to the beam waist. In fact, in the case of a physically realizable horn, the waist occurs at some negative phase slippage, and the phase slippage is only zero once the diverging beam has reached the aperture. In summary, the plot shows how the image of a diagonal horn propagates in a quasioptical system; it does not show how the scale size propagates; the scale size is determined by the waist. The plot is very easy to use: one simply moves along the beam calculating the total phase slippage,

$$
\phi=\tan \frac{Z}{Z c},
$$

where $Z c$ is the confocal distance [8], as one proceeds. Images come and go as the phase slippage accumulates, and at any point one can use the total phase slippage to determine the normalized radius at which a given level of truncation occurs. The actual physical radius is then calculated by multiplying the normalized radius by the beam width at that plane. Fig. 4 clearly shows that in the case of a diagonal horn it is necessary, in some regions, to clear the beam out to as much as 6 beam waists. In Table 1 , we show the phase slippage, and hence truncation loss, for each of the main components in the above optical system. Clearly, most of the problems are caused by $\mathrm{M} 2$ and the lens and window. In fact, it is the need to clear the beam of a diagonal horn out to large radii that makes it possible to use the system at frequencies as low as $200 \mathrm{GHz}$ with a corrugated horn.

One particular problem with diagonal horns is that it is impractical to clear the beam out to a loss better than about $1.5 \%$. It is a mistake, however, to think that if one has a cascade of truncating components the total loss is simply determined by combining the losses associated with the individual components. Obviously, if the beam does not diffract at the apertures, the 


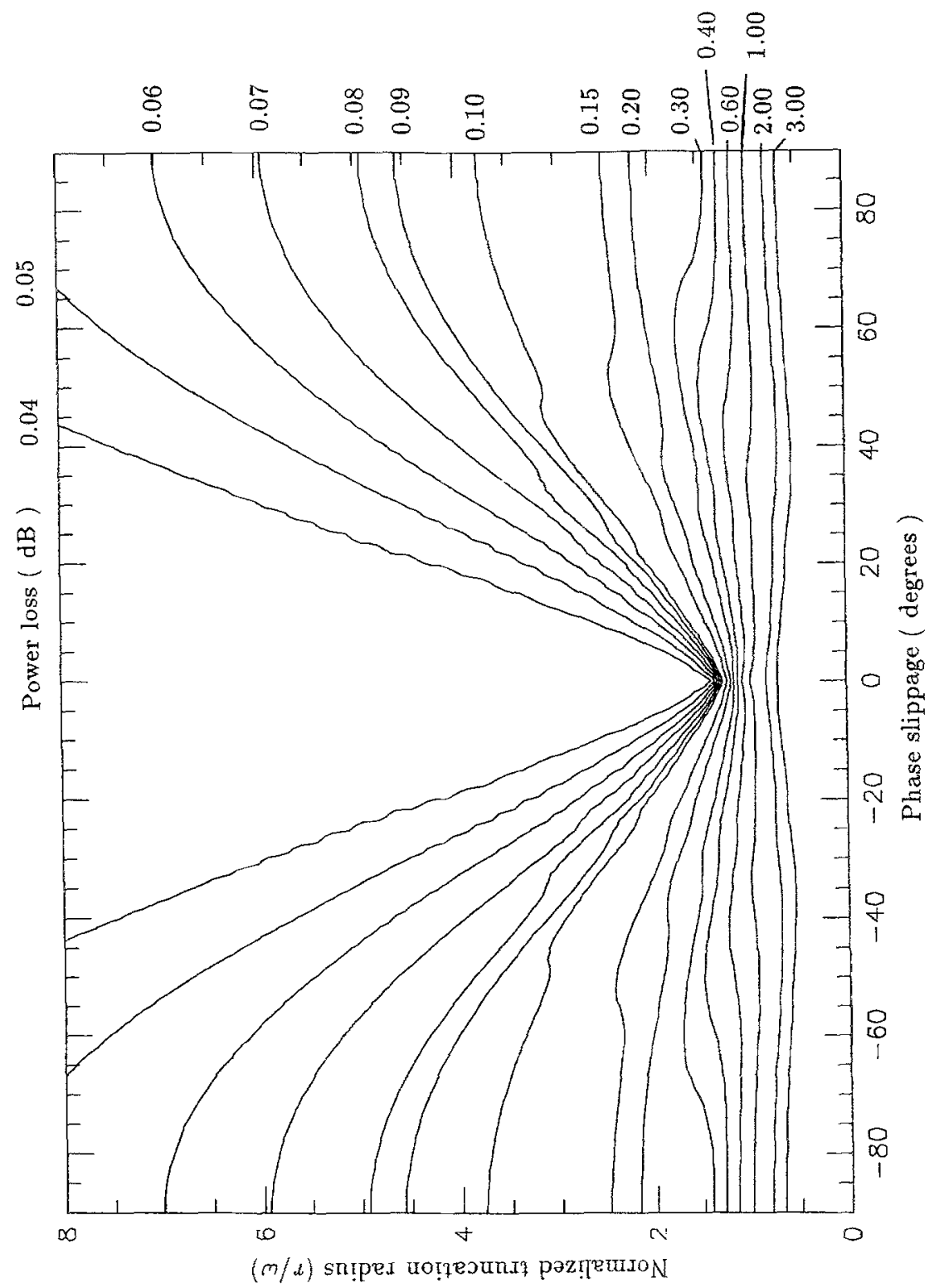

Figure 4: Contours of constant power loss $(\mathrm{dB})$ as a function of phase slipparse and normalized truncation for the co-polar beam of a diagonal horn. 


$\begin{array}{ccccccc}\text { Component } & \text { Separation } & \begin{array}{l}\text { Waist } \\ \text { size }\end{array} & \begin{array}{c}\text { Confocal } \\ \text { distance }\end{array} & \begin{array}{c}\text { Normalized } \\ \text { truncation }\end{array} & \begin{array}{l}\text { Phase } \\ \text { slippage }\end{array} & \text { Loss } \\ (\mathrm{mm}) & (\mathrm{mm}) & (\mathrm{mm}) & & \text { (Degrees) } & (\%)\end{array}$

SIGNAL PATH (400 GHz)

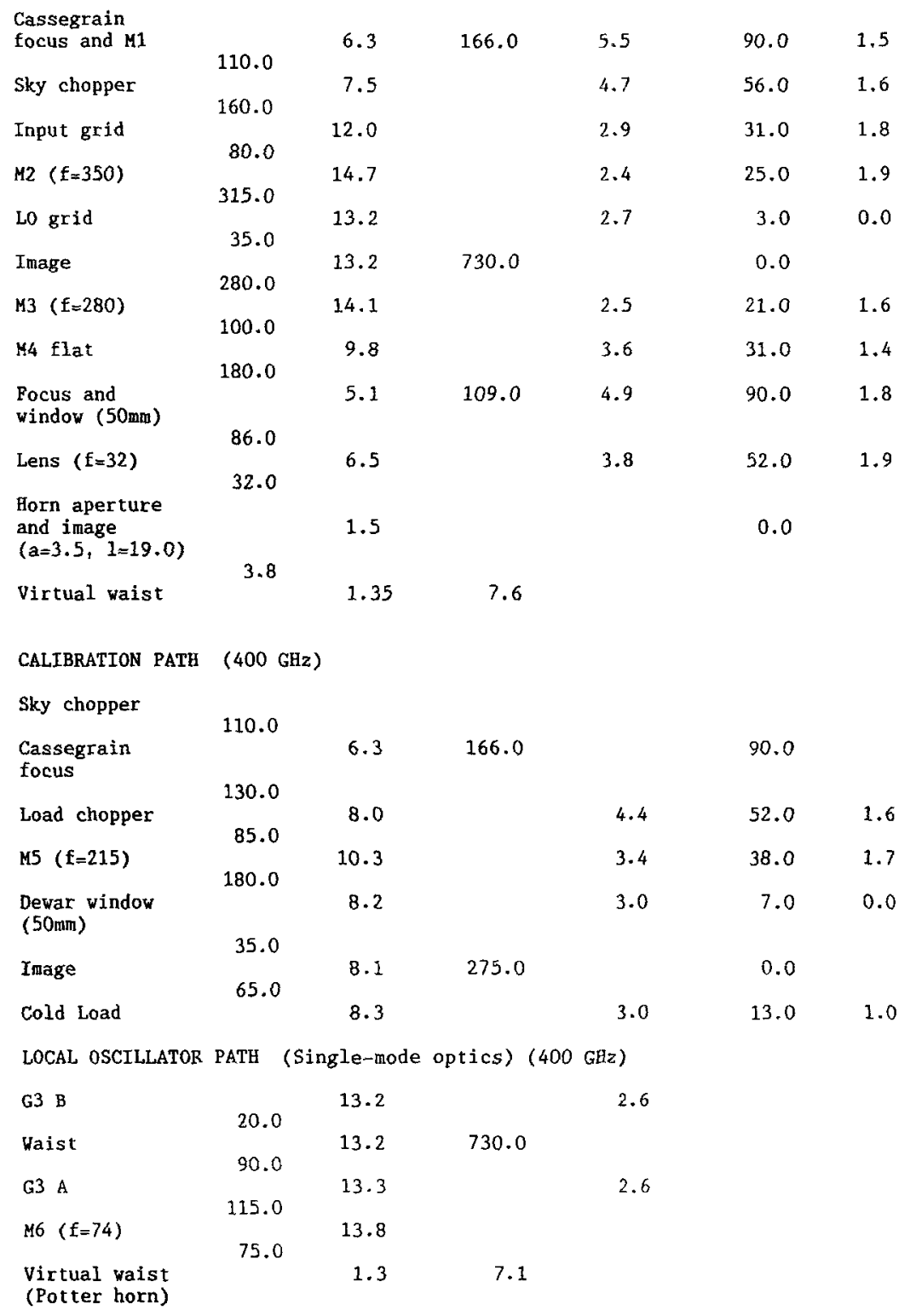

Table 1: Parameters of the optical system. 
total loss is simply given by the aperture with the greatest loss as it is not possible to lose power twice by truncating a beam at the same radius. If, on the other hand, truncation, causes the beam to diffract, then power may be lost at all of the apertures. Given that it is not possible to truncate the fields of a diagonal horn at a loss less than about $1.5 \%$, we were concerned about the degree to which truncation losses accumulate in our optics. To perform the full loss calculation one needs to determine how the beam changes as as it passes through each of the stops. We have used a scattering-parameter technique to propagate the beam through the optical system, and to calculate the real truncation loss at each of the apertures. These calculations are somewhat involved as it is necessary to expand the fields of the diagonal horn in terms of associated-Laguerre modes so that one can take advantage of the circular symmetry of an idealized stop. The details of these calculations will be presented in another paper, however, for the moment it is sufficient to say that the total loss turns out to be much smaller than the combined apparent losses of all of the components. In Fig. 5 we further demonstrate the scattering-parameter technique by showing the beam profiles at a number of planes in the system; of particular interest is the final beam that will result when the receiver is installed on the JCMT. Of course the truncation and beam profiles will be different when corrugated horns are used. In the case of the final beam we have assumed that the telescope behaves as a single long-focal-length optical component.

\section{Aberrations}

Another important consideration, as far as frequency independence is concerned, is the degree to which off-axis mirrors produce aberrations when they are used at frequencies away from the nominal design frequency. In the case of a lens whose axis coincides with the axis of propagation, the amplitude distribution of the beam on transmission through the lens is not significantly altered if the lens is thin and the focal ratio of the beam, $f / 2 \omega_{m}$, is large-- $\omega_{m}$ is the beam waist at the mirror and $f$ is the focal length. The effect of the lens can then be regarded purely as a phase-curvature transformation. Obviously, a focusing mirror can also be regarded as a phase transformer, however, in the case of an off-axis mirror, some amplitude distortion will occur even if the curvature of the mirror is relatively small [9]. In the case of the fundamental Gaussian mode, and assuming that the amplitude of the fundamental mode is close to unity, this aberration can be shown [9] to have the effect of scattering power primarily into a few high-order modes, with the amplitudes of the modes being of order $\left(\omega_{m} / 4 f\right)$ tan $i$, and the total fractional power scattered being $(1 / 8)\left(\left(\omega_{m} / f\right) \tan i\right)^{2}$, where $i$ is the angle of incidence of the beam at 

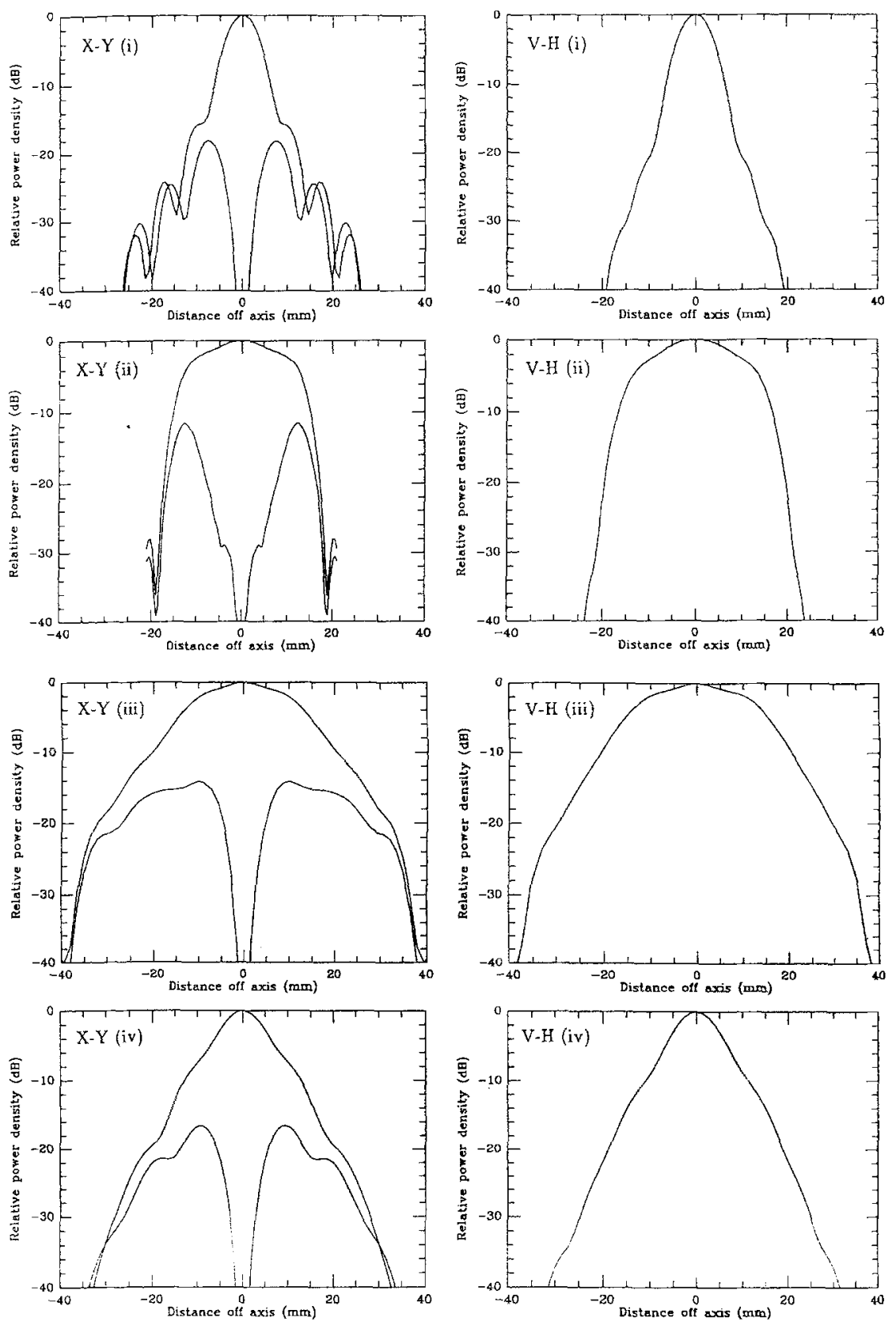

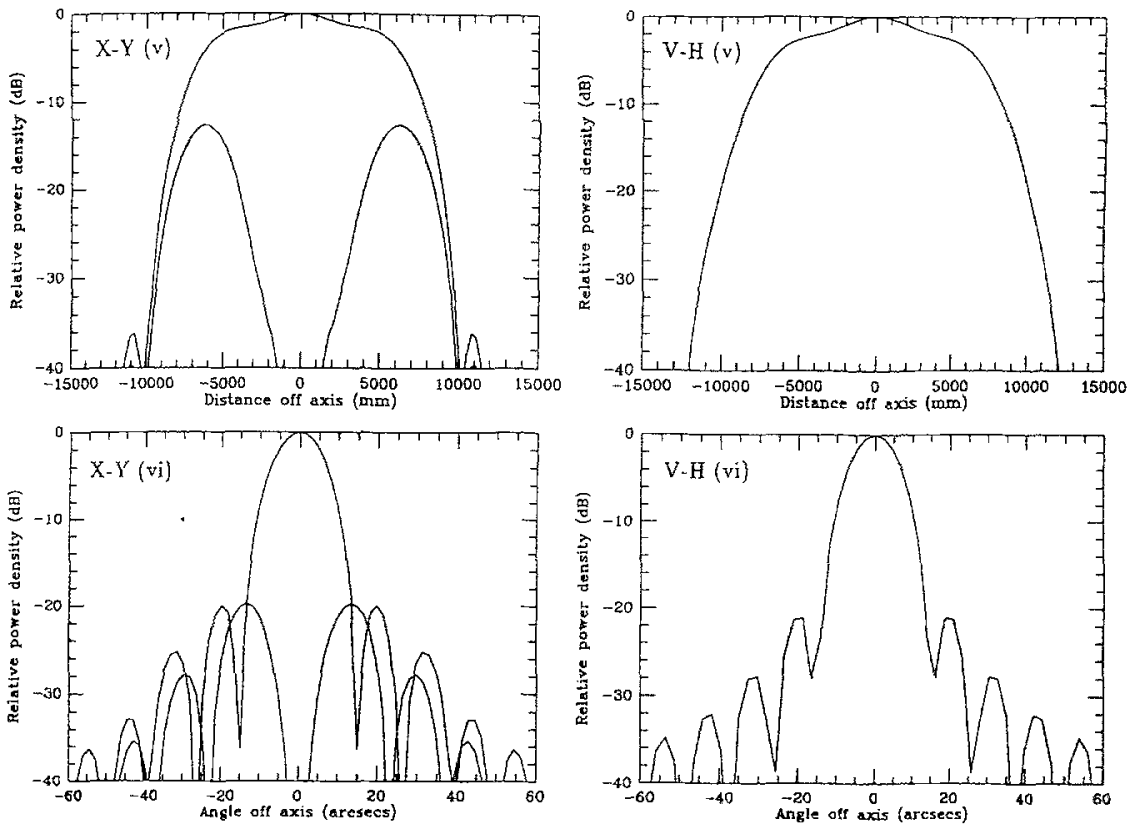

Figure 5: Beam profiles at various planes in the receiver; the two curves for the diagonal, $\mathrm{X}-\mathrm{Y}$, axis correspond to the co-polar and cross-polar fields. The diagonal horn does not have any cross-polar field in the vertical and horizontal, V-H, directions. The pairs of plots show the beam at the following positions: (i) the beam incident on the Dewar window, (ii) the beam at the first image of the horn, which is between the interferometers, (iii) the truncated beam transmitted by $M 2$, (iv) the beam at the focus of the telescope, and (vi) the beam that will result when the receiver is installed on the JCMT. 
the mirror surface. This aberration does not, in fact, placrs a severe limit on mirror configurations designed for Gaussian beams. The effect of amplitude distortion on the beam from a diagonal horn will be more complicated, nevertheless, we can get an good idea of system performance by applying the above calculations to the lowest-order Gaussian mode produced by the horn. In the case of our optics, M2 and M3, at the extremes of frequency, $200 \mathrm{GHz}$ and $900 \mathrm{GHz}$, the power lost from the fundamental is less than $0.05 \%$. This loss is very small because we have used long-focal-length mirrors throughout. It turns out that losses due to cross-polar scattering at the mirrors have the same functional form as the losses due to amplitude distortion, and therefore we can ignore cross-polarization losses also [9].

To ensure no phase aberration, the surface of an off-axis mirror must be a conic section of revolution, and the radii of curvature of the incident and reflected beams must match the distances to the two foci defined by the mirror surface. Since the radius of curvature of a beam is, in general, frequency dependent, broadband systems using off-axis mirrors may suffer from phase distortion at frequencies away from the nominal design frequency. We now summarize some important results concerning the severity of this problem. We model the effect of an off-axis mirror by using an extension of a technique already published [9]. In short, the reflected beam can be represented as though in transmission, by performing a reflection through the tangent plane at the beam-axis/mirror intersection point. The local Cartesian coordinate frame is defined, in the usual way, with its origin coinciding with the centre of the mirror on the beam axis. Let $R_{1}$ and $R_{2}$ be the distances from the centre of the mirror to the two foci; the focal length of the mirror is then given by the expression $f=R_{1} R_{2} /\left(R_{1}+R_{2}\right)$. The mirror is thus being modelled as an offaxis thin lens of refractive index -1 , with the phase-curvature transformation occurring at the tangent plane to the two "lens" surfaces. The technique of treating reflecting surfaces as refracting surfaces with negative refractive indices is well known in classical optics [10]. After some work, the phase transformation at the mirror tangent plane can be shown to be

$$
\Delta \phi(x, y, z) \approx \frac{\pi\left(x^{2}+y^{2}\right)}{\lambda}\left[\frac{1}{\left(R_{1}+z\right)}+\frac{1}{\left(R_{2}+z\right)}\right]
$$

Indeed, this expression ensures that there is no phase aberration provided $R_{1}=R_{i}(0)$, the phase radius of curvature of the incident beam at $z=0$, and $R_{2}=-R_{*}(0)$, the phase radius of curvature of the reflected beam at $z=0$. In a broadband system, there may be a significant mismatch at frequencies away from the nominal design frequency. In that case, $R_{i}(0) \neq R_{1}$; in fact, $1 / R_{o}(0)=1 / R_{i}(0)-1 / f$. It is straightforward to show that the distorted 
phase curvature of the output beam can be expressed as

$\Delta \phi_{a b}(x, y, z) \approx \frac{\pi\left(x^{2}+y^{2}\right)(x \tan i)}{\lambda}\left[\left(\frac{1}{R_{i}(0)^{2}}-\frac{1}{R_{1}^{2}}\right)+\left(\frac{1}{R_{0}(0)^{2}}-\frac{1}{R_{2}^{2}}\right)\right]$.

Thus, since the phase error is proportional to $\left(x^{2}+y^{2}\right) x$, the aberration can be classified as coma. In terms of a modal description, power in the fundamental mode will be scattered into the $\psi_{1,0}, \psi_{1,2}$, and $\psi_{3,0}$ higher-order Gaussian-Hermite modes. The total fractional power scattered will be of order

$$
L \approx\left[\frac{\pi \omega_{m}^{3}(0) \tan i}{\lambda}\left[\left(\frac{1}{R_{i}(0)^{2}}-\frac{1}{R_{1}^{2}}\right)+\left(\frac{1}{R_{o}(0)^{2}}-\frac{1}{R_{2}^{2}}\right)\right]\right]^{2} .
$$

If this term is negligible, the coma spot size is much less than the size of the output waist, and the phase distortions are unimportant. Once again, although the above description is strictly only valid for a single mode system, which is not the case when diagonal horns are used, we have applied the above criteria to our optical design. Taking the nominal design frequency to be $475 \mathrm{GHz}$, we find that, over the frequency range $200 \mathrm{GHz}$ to $900 \mathrm{GHz}$, the total amount of power scattered out of the fundamental is around $0.1 \%$. Hence once again, we come to the conclusion that the aberrations are insignificant.

\section{Conclusions}

We have designed a very broadband quasioptical system for a submillimetrewave radio astronomy receiver. The system combines the functions of imaging, local oscillator injection, sideband filtering, and calibration, and in principle, it can be used anywhere in the frequency range $200 \mathrm{GHz}$ to $900 \mathrm{GHz}$ without changing horns or mirrors. The system can be used in either a dualpolarization mode, where one observes a single spectral line in two orthogonal linear polarizations simultaneously, or in a dual-frequency mode, where one observes two spectral lines at different frequencies simultaneously:

At frequencies above $400 \mathrm{GHz}$, the optics have been designed to accommodate diagonal horns. Diagonal horns are much easier to manufacture than corrugated horns, but they are less tolerant of bad optical design. We have, therefore, studied the optical design of our receiver in much more detail than would usually be the case. For example, we have developed a imaging technique to determine the losses that occur when the beam from a diagonal horn is reimaged and truncated, and we have used a scattering-parameter technique to determine the losses that occur in the system as a whole. We have 
also used the scattering-parameter technique to calculate the beam shape that will result when the receiver in installed on the JCMT. Finally we have investigated the importance of the aberrations caused by using off-axis mirrors at frequencies a long way from the nominal design frequency, and we have shown that, for most long-focal-length systems, these aberrations can be ignored. Perhaps one of the most important conclusions of this paper is that it should be possible to make complicated quasioptical systems that have several octaves of bandwidth.

\section{Acknowledgements}

The authors would like to thank Robert Baldwin for producing the diagrams shown in this paper, and EOLAS and the British Council for financing travel.

\section{References}

[1] J.M. Payne, "Millimetre and submillimetre wavelength radio astronomy," Proc. IEEE, vol. 77, pp. 993-1017, July 1989.

[2] A.R. Kerr and S.-K. Pan, "Some recent developments in the design of SIS mixers," Int. J. Infrared Millimeter Waves, vol. 11, pp. 1169-1187, Oct. 1990.

[3] A.G. Murray, A.M. Flett, G. Murray, and P.A.R. Ade, "High efficiency half-wave plates for submillimetre polarimetry," Infrared Physics, vol. 33, pp. 113-125, 1992.

[4] J.B. Shapiro and E.E. Bloemhof, "Fabrication of wire-grid polarizers and dependence of submillimeter-wave optical performance on pitch uniformity," Int. J. Infrared Millimeter Waves, vol. 11, pp. 973-980, Aug. 1990.

[5] R. Padman, J.A. Murphy, and R.E. Hills, "Gaussian mode analysis of Cassegrain antenna efficiency," IEEE Trans. Antennas Propagat., vol. AP-35, pp. 1093-1103, Oct. 1987.

[6] S. Withington and J.A. Murphy, "Analysis of diagonal horns through Gaussian-Hermite modes," IEEE Trans. Antennas Propagat., vol. AP40, pp. 198-206, 1992.

[7] J.A. Murphy, A. Egan, and S. Withington, "Truncation in beam waveguides," To be published. 
[8] P.F. Goldsmith, "Quasioptical techniques at millimeter and submillimeter wavelengths,"in Infrared and Millimeter Waves, vol. 6, New York: Academic, pp. 277-343, 1982.

[9] J.A. Murphy, "Distortion of a simple Gaussian beam on reflection from off-axis ellipsoidal mirrors," Int. J. Infrared Millimeter Waves, vol. 8, pp. 1165-1187, 1987.

[10] M. Born and E. Wolf, "Principles of optics," Pergamon, Oxford, 1980. 